\title{
Dialysis Dose and Intradialytic Hypotension: Results from the HEMO Study
}

\author{
Finnian R. Mc Causland ${ }^{a, c}$ Steven M. Brunelli ${ }^{a-c}$ Sushrut S. Waikar ${ }^{a, c}$ \\ ${ }^{a}$ Renal Division and ${ }^{b}$ Division of Pharmacoepidemiology and Pharmacoeconomics, Department of Medicine, \\ Brigham and Women's Hospital and 'Harvard Medical School, Boston, Mass., USA
}

\section{Key Words}

Intradialytic hypotension - Urea reduction - Osmolality . Hemodialysis · HEMO study

\begin{abstract}
Background: Intradialytic hypotension (IDH) is common and is associated with increased morbidity and mortality in chronic hemodialysis patients. A higher dialysis 'dose' may generate transient intradialytic osmotic gradients, predisposing to intracellular fluid shifts and resulting in hypotension. Study Design: We performed a post hoc analysis of the HEMO study, a multicenter trial that randomized chronic hemodialysis patients to high versus standard Kt/ $\mathrm{V}$ and higher versus lower membrane flux. In order to achieve dose targets, per protocol, adjustments were made in membrane efficiency, blood flow or dialysate flow before changing session length. Detailed hemodynamic and urea kinetic modeling data were abstracted from 1,825 individuals. The primary outcome was the occurrence of hypotensive events necessitating clinical intervention (saline infusion, lowering of ultrafiltration rate or reduced blood flow). Results: Intradialytic hypotensive events occurred more frequently in the higher-Kt/V group (18.3 vs. $16.8 \% ; \mathrm{p}<0.001$ ). Participants randomized to higher-target $\mathrm{Kt} / \mathrm{V}$ had a greater adjusted risk of IDH than those randomized to standard Kt/V [odds ratio (OR) 1.12; 95\% confidence interval (CI) 1.01-1.25]. Higher vs.
\end{abstract}

lower dialyzer mass transfer-area coefficient for urea and rate of urea removal were associated with greater adjusted odds of IDH (OR 1.15; 95\% Cl 1.04-1.27 and OR 1.05; 95\% Cl 1.04$1.06 \mathrm{per} \mathrm{mg/dl} / \mathrm{h}$, respectively). Conclusions: Higher dialysis dose, at relatively constrained treatment times, may associate with an increased risk of IDH. These findings support the possibility that rapidity of intradialytic reductions in plasma osmolality may play an important role in mediating hemodynamic instability during dialysis.

(c) 2013 S. Karger AG, Basel

\section{Introduction}

Intradialytic hypotensive events are a common complication of maintenance hemodialysis, affecting up to one third of chronic dialysis treatment sessions [1-3]. Intradialytic hypotension (IDH) can be defined as an abrupt decline in blood pressure that causes symptoms and/or requires an intervention $[4,5]$. IDH has been associated with many adverse clinical events, including myocardial stunning [6], cerebral atrophy [7] and increased mortality [8]. Predisposing factors include intrinsic patient-related

Portions of this work were presented in the form of an abstract at the 2012 annual meeting of the American Society of Nephrology in San Diego.

\section{KARGER}

E-Mail karger@karger.com www.karger.com/ajn
(C) 2013 S. Karger AG, Basel

0250-8095/13/0385-0388\$38.00/0
Finnian Mc Causland, MB, MMSc

MRB-4, Brigham and Women's Hospital

Boston, MA 02446 (USA)

E-Mail fmccausland@ partners.org 
factors such as the presence of autonomic neuropathy [9], abnormal cardiac reserve [10] and reduced venous compliance [11] as well as potentially modifiable treatmentrelated parameters such as ultrafiltration (UF) profiling [12] and changes in serum calcium concentration [13].

The rapidity of removal of urea, sodium and other osmotically active substances from the intravascular compartment, in conjunction with delayed re-equilibration from intracellular compartments, may result in a transient decline in plasma osmolality and intravascular volume depletion secondary to transcellular movement of water. This may predispose to the development of IDH [14]. In support of this concept, investigators have reported that administration of hypertonic glucose or hypertonic saline ameliorates intradialytic blood pressure decline and other dialysis-associated symptoms [15]. In addition, the use of sodium modeling algorithms has been associated with improved hemodynamic parameters in patients with a prior history of IDH [4].

In the HEMO study [16], a multicenter, randomized trial in maintenance hemodialysis, patients were randomized to higher versus standard Kt/V and higher versus lower membrane flux. Study investigators were instructed to increase blood flow $(\mathrm{Qb})$, dialysate flow $(\mathrm{Qd})$ and filter surface area before increasing the session length in order to achieve higher Kt/V targets. Therefore, we used the randomized dose assignment to examine the effect of rapid osmotic shifts during hemodialysis and tested the hypothesis that higher dialysis dose associates with a greater risk of IDH.

\section{Methods}

\section{Study Design and Population}

The study protocol was deemed exempt by the Partners Healthcare Institutional Review Board. All data were abstracted from the HEMO study with the permission of the National Institute of Diabetic and Digestive and Kidney diseases. The design of the HEMO study has been previously reported $[16,17]$. Briefly, HEMO was a prospective, multicenter, randomized clinical trial of low-flux versus high-flux membranes and high versus standard dialysis dose (target single-pool Kt/V 1.65 vs. 1.25; approx. equivalent to urea reduction ratios of 75 vs. $65 \%$ ) among prevalent adult subjects receiving thrice-weekly in-center hemodialysis. Exclusion criteria included a baseline serum albumin $<2.6 \mathrm{~g} / \mathrm{dl}$, residual urea clearance of $\geq 1.5 \mathrm{ml} / \mathrm{min} / 35$ liters of urea distribution volume, inability to consistently achieve an equilibrated $\mathrm{Kt} / \mathrm{V}$ of $>1.3$ or the presence of end-stage comorbid conditions other than kidney failure. Of the 1,846 HEMO study participants, we excluded those who did not have available hemodynamic and kinetic modeling data after randomization $(n=20)$; our final cohort consisted of 1,825 individuals and 62,095 hemodialysis treatment sessions.

\section{Exposures and Outcomes}

The primary analysis examined the HEMO study randomized $\mathrm{Kt} / \mathrm{V}$ assignment (higher vs. lower) as the exposure of interest. In secondary analyses, the association of individual components of dialysis 'dose' with IDH was examined [Qb, Qd, session length, dialyzer mass transfer-area coefficient for urea $\left(\mathrm{KoA}_{\text {urea }}\right)$ and rate of decline in plasma blood urea nitrogen (BUN)]. In vitro $\mathrm{KoA}_{\text {urea }}$ values were previously obtained for 22 HEMO study-approved membranes at a Qd of $800 \mathrm{ml} / \mathrm{min}$ [18]. The rate of decline in plasma BUN was calculated using BUN measurements as: predialysis BUN to postdialysis BUN/session length, after exclusion of the top and bottom $1 \%$ of individual BUN measurements as outliers. Data for all urea-based exposure calculations were abstracted from monthly 2-sample BUN modeling sessions, where post-BUN was taken $15 \mathrm{~s}$ (from line disconnect) or $20 \mathrm{~s}$ (from sampling port) after dialysis inlet slowing.

The primary outcome was the occurrence of an intradialytic hypotensive event, defined as a hypotensive episode requiring either saline infusion, lowering of the UF rate or reduction in Qb. This was a prespecified outcome of the HEMO study that was assessed and recorded on a monthly basis during monitored hemodialysis sessions by trained study personnel. In sensitivity analyses, an alternative definition of IDH was examined: decline in systolic blood pressure (SBP) of $\geq 50 \mathrm{~mm} \mathrm{Hg}$ with headache, cramps or vomiting and requirement for an intervention (lowering of Qb, UF or saline infusion).

\section{Study Data}

All study data in the HEMO study were obtained by subject interview, chart review and self-reported questionnaires. Demographic data including sex, race, and age were recorded at baseline [taken as the time of the first kinetic modeling session after randomization for the purposes of this study; median time after randomization of 20 days (IQR 13-28)]. Prerandomization kinetic modeling sessions were used to assess the baseline incidence of IDH. Other variables of interest included comorbidities, i.e. diabetes, ischemic heart disease, peripheral vascular disease, congestive heart failure and arrhythmia (recorded annually), dialysis treatment and hemodynamic parameters (recorded monthly) and laboratory parameters (recorded 6-monthly). Comorbidities were graded on the Index of Coexisting Disease (ICED) scale. Analytically, these were dichotomized ( 0 if ICED score $=0$ and 1 if ICED score $\geq 1$ ) except for congestive heart failure, where more granular categorization was considered ( 0 if ICED score $=0,1$ if ICED score $=1$ and 2 if ICED score $\geq 2$ ) [19].

\section{Statistical Analysis}

Continuous variables were examined graphically and recorded as means ( \pm standard deviations) for normally distributed data or medians (with IQRs) for nonnormally distributed data. Comparisons were made using the Student t test, the Wilcoxon rank sum test, analysis of variance or the Kruskal-Wallis test where appropriate. Categorical variables were examined by frequency distribution, recorded as proportions and comparisons were made using the $\chi^{2}$ test. Initially, unadjusted generalized linear regression models (using a binomial distribution and logit link function) were fit [20] to assess the relationship between randomized Kt/V group and hypotensive events (Yes/No). These models used clustered variance estimates to account for nonindependence of covariates within subject. Subsequently, adjustment was made for random- 
ized HEMO study flux assignment and baseline prespecified covariates as done in the original published HEMO analyses (age, sex, race, vintage, diabetes, index of coexistent disease score and serum albumin) [16]; in our analyses, ICED score was considered as a categorical variable $(0,1,2$ or 3$)$. In secondary analyses, the associations of categories of Qb $(\leq 250,251-350,351-450$ and $>450$ $\mathrm{ml} / \mathrm{min})$, Qd ( $\leq 500,501-799$ and $\geq 800 \mathrm{ml} / \mathrm{min}$ ), session length $(\leq 180,181-209,210-239$ and $\geq 240 \mathrm{~min}), \mathrm{KoA}_{\text {urea }}(<1,000$ and $\geq 1,000 \mathrm{ml} / \mathrm{min}$ ) and rate of decline in plasma BUN ( $\mathrm{mg} / \mathrm{dl} / \mathrm{h})$ with IDH were each individually examined using unadjusted and adjusted generalized linear models. For these analyses, exposure variables and covariates were considered as the most proximate value preceding each kinetic modeling session. Multivariable models (model 1) included terms for HEMO study flux assignment, age, race, sex, postdialysis weight, height, access, predialysis SBP, ischemic heart disease, congestive heart failure, diabetes, peripheral vascular disease, arrhythmia, predialysis serum sodium, UF requirement, serum albumin, creatinine, phosphorus and bicarbonate. As the prognostic significance of body size may differ according to gender, 2-way sex-by-postdialysis-weight cross-product terms were included. In model 2 , the association of $\mathrm{KoA}_{\text {urea }}$ (model 2a) and rate of decline in plasma BUN (model 2b) with IDH were each individually examined after adjustment for categories of $\mathrm{Qb}$, Qd and session length, in addition to the same covariates as model 1 (categories of session length were removed from the list of covariates in model $2 \mathrm{~b}$ due to collinearity). Covariates for all models were selected on the basis of clinical and biological plausibility, without the use of probabilistic selection criteria. Effect modification of randomized Kt/V group on the basis of flux (high vs. low) assignment and predialysis SBP was examined for (and excluded) via the inclusion of 2-way cross-product terms.

Nominal 2 -sided $p$ values of $<0.05$ were considered statistically significant. Analyses were performed using STATA 10.0MP (College Station, Tex., USA).

\section{Results}

The primary cohort consisted of 1,825 individuals and 62,095 unique hemodialysis sessions. Mean age was $57.8 \pm 14.1$ years, $62.6 \%$ were black and $44.7 \%$ were diabetic. Consistent with other reports from the HEMO study, there was a slightly greater proportion of patients with peripheral vascular disease in those randomized to the higher-Kt/V group [21]; otherwise, no significant baseline differences between randomized groups were evident (table 1). Those in the higher-Kt/V group had significantly longer session length, higher $\mathrm{Qb}$ and higher $\mathrm{Qd}$ compared with those in the lower Kt/V group (table 2). There were no significant differences in preintervention rates of IDH between randomized Kt/V groups.

At baseline, those with a greater rate of decline in plasma BUN were more likely to be female, not black, of lesser postdialysis weight and have less peripheral vascular disease but have greater serum albumin and creatinine. In addition, they were more likely to have greater UF volume, shorter session length, lower $\mathrm{Qb}$ and higher Qd (online suppl. tables A, B; for all online suppl. material, see www.karger.com/doi/10.1159/000355958).

\section{HEMO Study Target Kt/V Assignment}

In the higher-Kt/V group, $18.3 \%$ of sessions were complicated by hypotensive events, compared with $16.8 \%$ of the lower-Kt/V group ( $<<0.001$; table 3$)$. The mean intradialytic decline in SBP in sessions with IDH was $52.9 \pm$ $27.5 \mathrm{~mm} \mathrm{Hg}$, compared with $29.3 \pm 22.0 \mathrm{~mm} \mathrm{Hg}$ in those without ( $\mathrm{p}$ difference $<0.001$ ).

In patient-level analyses, using generalized linear models, the unadjusted and adjusted odds of experiencing a hypotensive event were $11 \%$ higher [odds ratio (OR) $1.11 ; 95 \%$ confidence interval (CI) $0.99-1.24 ; \mathrm{p}=0.07]$ and $12 \%$ higher (OR 1.12; 95\% CI 1.01-1.25; $\mathrm{p}=0.04$ ), respectively, for the higher-Kt/V vs. the lower-Kt/V group. There was no evidence for effect modification according to randomized flux target ( $p$ interaction $=0.19$ ).

In sensitivity analyses, a more restrictive definition of IDH was considered (decline in SBP of $50 \mathrm{~mm} \mathrm{Hg}$, with symptoms or need for intervention). In this model, higher Kt/V remained associated with greater odds for IDH (OR 1.10; 95\% CI 1.00-1.21; $\mathrm{p}=0.049$ ).

\section{Session Length, Blood Flow and}

Unadjusted and adjusted models were fit (individually for session length, $\mathrm{Qb}, \mathrm{Qd}$ and $\mathrm{KoA}_{\text {urea }}$ ) to examine which of these contributors to dialysis dose were independently associated with IDH (table 4). Compared with the reference for each exposure, there was no evidence for an association of greater session length, Qb, Qd or KoA $\mathrm{Area}_{\text {ure }}$ with a greater risk for IDH. In fact, greater $\mathrm{Qb}$ appeared to be associated with less risk of IDH in adjusted models. However, upon additional adjustment for Qb, Qd and session length in model $2 \mathrm{a}$, higher (vs. lower) $\mathrm{KoA}_{\text {urea }}$ became significantly associated with a greater risk of IDH (OR 1.15; 95\% CI 1.04-1.27).

\section{Rate of Decline in Plasma BUN}

In light of the observation that higher $\mathrm{KoA}_{\text {urea }}$ associates with greater risk of IDH in fully adjusted models, we revisited our original hypothesis that rapid solute removal may generate temporary osmolar gradients and predispose to IDH. Therefore, we considered the rate of decline in plasma BUN as an alternative metric to be examined. In patient-level analyses, using generalized linear models, the unadjusted and adjusted odds (model 1) of experiencing IDH were $4 \%$ higher (OR 1.04; 95\% CI 1.03-1.06) and 
Table 1. Baseline characteristics of the total study cohort according to target Kt/V assignment

\begin{tabular}{|c|c|c|c|c|}
\hline & \multirow{2}{*}{$\begin{array}{l}\text { Total cohort } \\
(\mathrm{n}=1,825)\end{array}$} & \multicolumn{2}{|l|}{$\mathrm{Kt} / \mathrm{V}$ assignment } & \multirow[t]{2}{*}{$\mathrm{p}^{\mathrm{a}}$} \\
\hline & & standard $(n=915)$ & higher $(n=910)$ & \\
\hline Male, \% & 43.8 & 44.0 & 43.6 & 0.86 \\
\hline Age, years & $57.8 \pm 14.1$ & $58.0 \pm 14.0$ & $57.6 \pm 14.1$ & 0.51 \\
\hline Black, \% & 62.6 & 64.2 & 61.1 & 0.18 \\
\hline Vintage, years & $2.3(1.1-4.8)$ & $2.4(1.2-5.0)$ & $2.2(1.1-4.6)$ & 0.16 \\
\hline Diabetes, \% & 44.7 & 44.7 & 44.6 & $>0.9$ \\
\hline Ischemic heart disease, $\%$ & 39.3 & 39.9 & 38.7 & 0.60 \\
\hline Congestive heart failure, $\%$ & & & & 0.88 \\
\hline Mild & 27.9 & 27.5 & 28.3 & \\
\hline Moderate/severe & 11.5 & 11.8 & 11.2 & \\
\hline Peripheral vascular disease, $\%$ & 25.6 & 23.5 & 27.7 & 0.04 \\
\hline Arrhythmia, \% & 30.8 & 32.4 & 29.3 & 0.16 \\
\hline ICED score & $2.0(1.0-3.0)$ & $2.0(1.0-3.0)$ & $2.0(1.0-3.0)$ & 0.41 \\
\hline Predialysis SBP, mm Hg & $152.1 \pm 26.3$ & $152.6 \pm 26.6$ & $151.6 \pm 26.0$ & 0.42 \\
\hline Postdialysis weight, kg & $69.4 \pm 14.8$ & $69.9 \pm 14.9$ & $68.9 \pm 14.7$ & 0.15 \\
\hline UF requirement, liters & $2.9 \pm 1.3$ & $2.9 \pm 1.3$ & $2.9 \pm 1.3$ & 0.32 \\
\hline $\mathrm{UFR}, \mathrm{ml} / \mathrm{kg} / \mathrm{h}$ & $12.2 \pm 5.6$ & $13.1 \pm 5.8$ & $11.3 \pm 5.3$ & $<0.001$ \\
\hline Access, $\%$ & & & & 0.86 \\
\hline AVG & 59.9 & 59.8 & 60.1 & \\
\hline AVF & 33.7 & 33.5 & 33.9 & \\
\hline Catheter & 6.4 & 6.7 & 6.0 & \\
\hline Serum sodium, mmol/l & $138.2 \pm 3.9$ & $138.3 \pm 4.0$ & $138.1 \pm 3.9$ & 0.34 \\
\hline Serum albumin, g/dl & $3.8 \pm 0.4$ & $3.8 \pm 0.4$ & $3.8 \pm 0.4$ & $>0.9$ \\
\hline Serum creatinine, mg/dl & $10.3 \pm 2.9$ & $10.3 \pm 2.8$ & $10.3 \pm 3.0$ & 0.89 \\
\hline Serum phosphorus, mg/dl & $5.8 \pm 1.9$ & $5.8 \pm 1.9$ & $5.8 \pm 1.9$ & 0.81 \\
\hline Serum bicarbonate, $\mathrm{mmol} / \mathrm{l}$ & $21.4 \pm 3.6$ & $21.4 \pm 3.7$ & $21.5 \pm 3.4$ & 0.67 \\
\hline
\end{tabular}

$\mathrm{AVF}=$ Arteriovenous fistula; $\mathrm{AVG}=$ arteriovenous graft; UFR = ultrafiltration rate.

${ }^{a} \mathrm{p}$ value for difference; significance testing was by Student $\mathrm{t}$ test or Wilcoxon rank sum tests for continuous variables or $\chi^{2}$ test for categorical variables.

Table 2. Baseline dialysis session characteristics of the total study cohort according to target Kt/V assignment

\begin{tabular}{lllll}
\hline & $\begin{array}{l}\text { Total cohort } \\
(\mathrm{n}=1,825)\end{array}$ & Kt/V assignment & $\mathrm{p}^{\mathrm{a}}$ \\
\cline { 3 - 4 } & & standard $(\mathrm{n}=915)$ & higher $(\mathrm{n}=910)$ & \\
\hline Session length, $\mathrm{min}$ & $210(180-225)$ & $185(175-210)$ & $215(200-240)$ & $<0.001$ \\
Prescribed Qb, ml/min & $400(300-450)$ & $350(300-400)$ & $400(400-450)$ & $<0.001$ \\
Prescribed Qd, $\mathrm{ml} / \mathrm{min}$ & $700(500-800)$ & $600(500-800)$ & $800(600-800)$ & $<0.001$ \\
KoA $_{\text {urea }} \geq 1,000 \mathrm{ml} / \mathrm{min}, \%$ & 43.6 & 36.3 & 51.1 & $<0.001$ \\
\hline
\end{tabular}

${ }^{a} \mathrm{p}$ value for difference; significance testing was by Wilcoxon rank sum test.

5\% higher (OR 1.05; 95\% CI 1.04-1.06), respectively, per unit increase in the rate of decline in plasma BUN (mg/ $\mathrm{dl} / \mathrm{h}$ ). Of note, this association was independent of Qb, Qd and UF volume (the latter also being significantly associated with IDH (OR 1.05; 95\% CI 1.02-1.09; Model 2).
In order to assess for possible nonlinear relationships, the rate of decline in plasma BUN was then considered as quartiles. A progressive increase in the proportion of sessions complicated by IDH was noted for increasing quartiles of rate of decline in plasma BUN (table 3). Of note, 
Table 3. Percentage of hemodialysis sessions complicated by IDH according to categories of exposure (unadjusted)

\begin{tabular}{|c|c|c|c|c|c|c|c|c|}
\hline & \multicolumn{3}{|c|}{ Randomized Kt/V group } & \multicolumn{5}{|c|}{ Quartile of rate of decline in plasma BUN } \\
\hline \multicolumn{9}{|c|}{ Percentage of sessions complicated by IDH } \\
\hline During intervention & 16.8 & 18.3 & $<0.001$ & 14.1 & 16.4 & 18.6 & 20.6 & $<0.001$ \\
\hline
\end{tabular}

Table 4. Association of individual components of dialysis dose with IDH

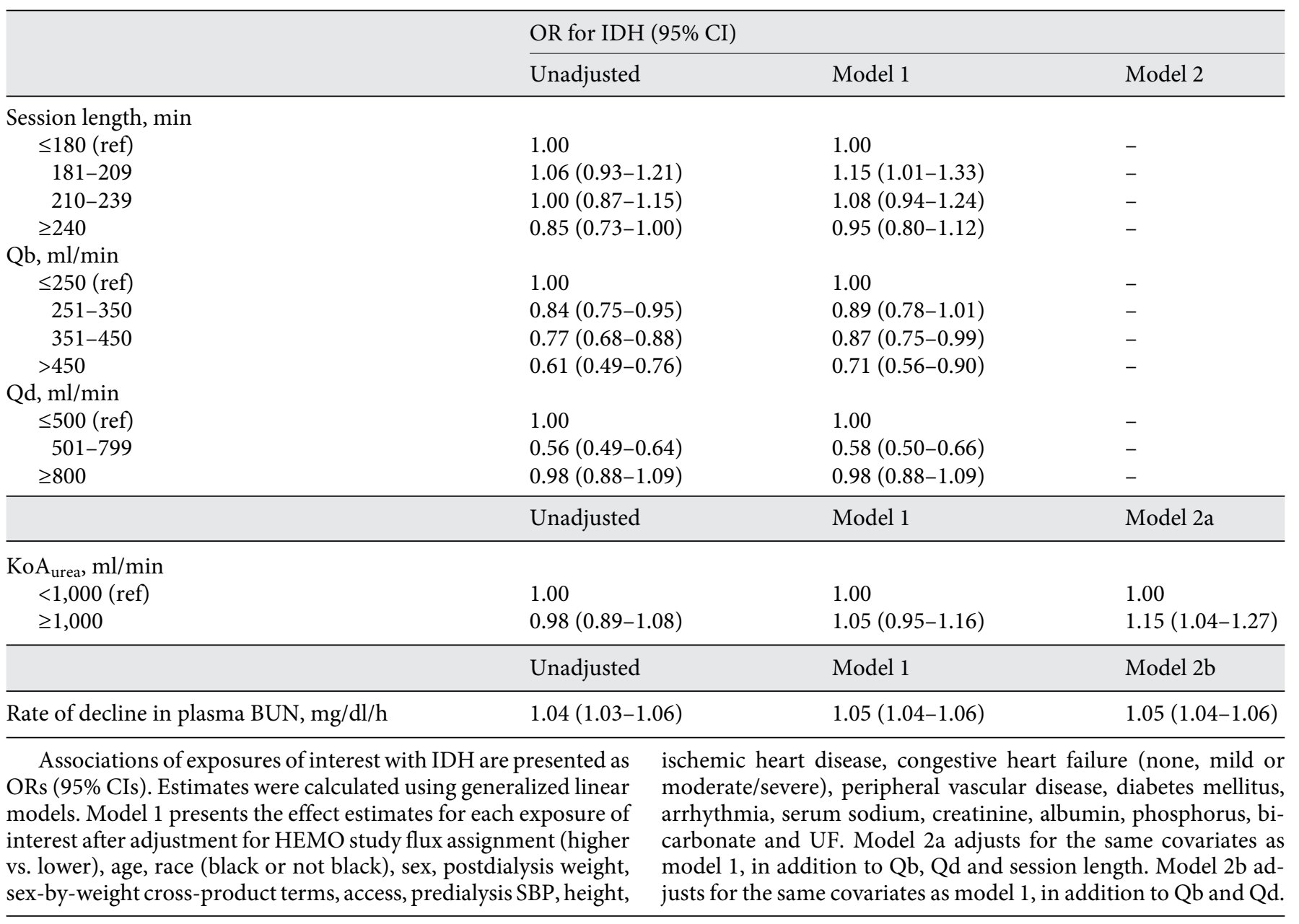

this pattern was also present in analyses of the preinterventional period. In adjusted analyses, a similar pattern was noted, with increasing odds for IDH associated with increasing quartiles of rate of decline in plasma BUN (fig. 1).

\section{Discussion}

Using data from the HEMO study, the largest study of dialysis dose to date, we found that patients randomized to a higher dose (target single-pool Kt/V 1.65 vs. 1.25) 


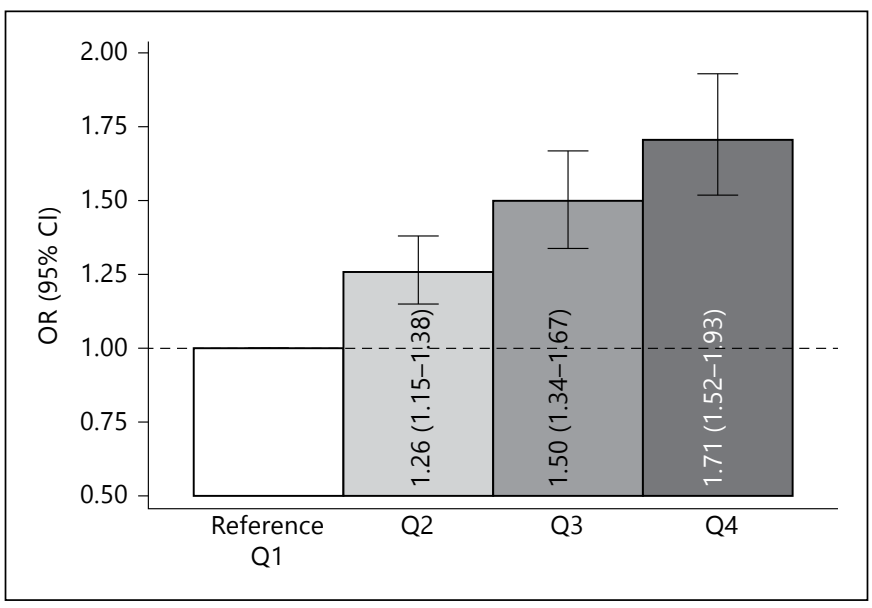

Fig. 1. The association between quartiles of rate of decline in plasma BUN and IDH. Associations between quartiles (Q; referent Q1) of rate of decline in plasma BUN and IDH are presented as ORs $(95 \% \mathrm{CI})$. Estimates were calculated using generalized linear models and adjusted for HEMO study flux assignment (higher vs. lower), session length $(\leq 180,>180, \geq 210$ and $\geq 240 \mathrm{~min})$, Qb $(\leq 250$, $250-349,350-449$ and $\geq 450 \mathrm{ml} / \mathrm{min})$, Qd $(0-500,501-800,>800$ $\mathrm{ml} / \mathrm{min}$ ), age, race (black and not black), sex, postdialysis weight, sex-by-weight cross-product terms, access, predialysis SBP, height, ischemic heart disease, congestive heart failure (none, mild and moderate/severe), peripheral vascular disease, diabetes mellitus, arrhythmia, serum sodium, creatinine, albumin, phosphorus, bicarbonate and UF requirement.

were more likely to experience IDH during hemodialysis. Notably, these findings occurred despite lower baseline UF rates ( 11.3 vs. $13.1 \mathrm{ml} / \mathrm{kg} / \mathrm{h}$ ) in the higher-Kt/V group, suggesting that other factors must be involved in the genesis of IDH.

Potential risk factors for IDH may include the presence of autonomic neuropathy [22] and diminished cardiac reserve [10]; however, there were no significant differences in the proportion of individuals with diabetes or heart failure according to higher vs. lower Kt/V groups in our study. We did note a higher proportion of individuals with peripheral vascular disease, as was reported in the HEMO study, but found no difference in effect estimates after additionally adjusting for peripheral vascular disease in multivariable models. Other possibilities include an impaired rate of plasma refilling [23], inflammatory cytokine release [24] and reduced venous compliance [11]. In our secondary analyses, we attempted to further dissect the relationship between 'dose' and IDH by examining the individual associations of session length, $\mathrm{Qb}$, $\mathrm{Qd}$ and $\mathrm{KoA}_{\text {urea }}$. These analyses were informative, in that we found no significant association of greater session length or Qd with IDH, and actually found lower odds of
IDH with greater $\mathrm{Qb}$ in adjusted models. A potential explanation for this observation may be confounding by patients who are able to achieve higher $\mathrm{Qb}$ being less likely to have vascular disease or diabetes, and thus able to tolerate higher flows. However, in line with our original hypothesis, we did note an association of greater $\mathrm{KoA}_{\text {urea }}$ and greater rate of decline in plasma $\mathrm{BUN}$ with greater odds for hypotension (independent of Qb and Qd; model 2), suggesting that this factor is an important determinant in the relationship between dialysis 'dose' and IDH.

Experimental data in animals $[25,26]$ and humans [27] support a role for rapid urea removal by hemodialysis in the generation of temporary osmotic gradients between body compartments. Others argue that the induction of temporary gradients for other molecules, including sodium, are more important [28], but there appears to be a consensus that these gradients contribute to many of the features of dialysis disequilibrium including cerebral edema, mental status changes, seizures and even death [29]. Several investigators have also noted an association between the intradialytic generation of transcellular osmotic gradients and IDH $[28,30,31]$. In order to achieve the higher randomized Kt/V target, the HEMO study investigators were encouraged to increase membrane size, Qd or Qb before increasing treatment time. Therefore, in effect, dialysis session lengths were relatively constrained, which likely increased the rapidity of temporary osmotic gradient generation between the intravascular and extravascular compartments. Our findings, based on the original HEMO targeted Kt/V randomization groups provide evidence for an association between the rapidity of intradialytic plasma osmolality decline and the development of hypotensive events. The absence of differences in the percentage of hypotensive events among preintervention dialysis sessions, according to randomized $\mathrm{Kt} / \mathrm{V}$ groups, provides supportive evidence that the higher-dose intervention is truly associated with a greater risk of IDH. In the secondary analyses, the association of increasing quartiles of rate of decline in plasma BUN (which are nonrandomized categories) with greater IDH was present for both preintervention and postintervention sessions. These analyses support the presence of a dose-response relationship between the achieved rate of decline in plasma BUN and risk of IDH, irrespective of the randomized trial intervention.

There are numerous reports detailing interventions aimed at minimizing the rate of osmolality decline that may be useful for the treatment of hypotensive-prone patients. Dialysis physicians initially increased the dialysate sodium concentration, finding that this reduced the fre- 
quency of dialysis-related symptoms [32], with subsequent studies demonstrating improved hemodynamic stability $[33,34]$. Prior reports of increased thirst and interdialytic weight gain tempered the initial enthusiasm of these findings [12,34], but more recently, the use of biofeedbackcontrolled sodium profiling appears to have less associated interdialytic weight gain [35]. Other methods include the administration of hypertonic mannitol and other hypertonic solutions $[15,30,36,37]$. In addition to transcellular fluid shifts due to osmotic changes, a rapid decline of intravascular osmolality may suppress the release of vasopressin, which has pressor-like effects on the vasculature; in support of this concept, infusion of vasopressin during hemodialysis has been associated with improved hemodynamic stability [38]. Given the association of IDH with numerous adverse outcomes (including access failure [39], cerebral atrophy [7], myocardial stunning [40] and death [8]) as well as the promise shown by previous small interventional studies, future research efforts focusing on manipulation of osmolality changes, without compromising clearance, may be fruitful in addressing the unacceptably high morbidity and mortality of our patients.

The major strength of this study is the utilization of the original randomized assignments from the HEMO study, which should minimize the presence of residual confounding in the primary analyses of targeted $\mathrm{Kt} / \mathrm{V}$. In this regard, although the HEMO study did not record potentially confounding variables such as dialysate sodium or dialysate temperature, it is likely that randomization produced a minimal imbalance according to these parameters between the targeted Kt/V groups. Furthermore, the HEMO study was performed at a time when the move away from lower sodium dialysate concentrations had already occurred [41]. Other strengths include the large and detailed collection of exposure and outcomes data over the complete duration of follow-up in the setting of a randomized controlled trial. Without the primacy of randomization in secondary analyses, multiple covariates had to be considered in the model-building process, leading to the possibility of residual confounding. There are theoretical concerns of dose-targeting bias in relation to the analysis of rate of decline in plasma BUN as the exposure of interest. For example, in the dose-mortality analyses of the HEMO study, significant associations between higher achieved dose and reduced mortality have been reported (at odds with the original intention-to-treat analyses demonstrating no advantage to higher vs. standard $\mathrm{Kt} / \mathrm{V}$ ) [42]. It is possible that individuals who were able to attain a higher achieved $\mathrm{Kt} / \mathrm{V}$ were healthier, more compliant and had other beneficial characteristics that could potentially confound the relationship between achieved dose and mortality. The associations we present are less likely to suffer from this bias, as we found that higher rates of urea removal (a metric of clearance, in which higher values may be interpreted as beneficial) were in fact associated with greater odds for hypotension. This is opposite to what we would expect if beneficial dose-targeting bias were an issue. However, on the other hand, a very high rate of decline in plasma BUN can also be achieved in malnourished individuals with low muscle mass or in those with shorter session lengths, who may be at risk for adverse outcomes. This may have contributed to the increased association with hypotensive events which we observed in these individuals. The HEMO study did not record the timing of hypotensive events, prohibiting a more granular assessment of the competing effects of solute removal rate and UF rates, which may be expected to predispose to hypotension at different time points during the dialysis procedure. However, we were able to confirm that both the rate of decline in plasma BUN and UF volume were independently associated with greater risk of IDH. Finally, participants in a randomized controlled setting may not be comparable to the wider chronic hemodialysis population, limiting the generalizability of our results.

In conclusion, a higher dialysis dose appears to increase the risk for intradialytic hypotensive events requiring a clinical intervention. In patients at risk of IDH, targeted strategies to reduce the rapidity of decline in plasma osmolality during hemodialysis may be beneficial. Potential interventions may include selection of lower efficiency membranes, extending the session length, selected use of higher dialysate sodium or infusion of osmotically active substances. While ensuring preservation of hemodialysis adequacy, future studies should assess the potential benefit of these interventions in prospective clinical trials, particularly in patients who suffer from repeated episodes of IDH.

\section{Acknowledgements}

We thank the HEMO study investigators and the National Institute of Diabetes and Digestive and Kidney Diseases (NIDDK) data repository for the data used in this study. The HEMO study was performed by the HEMO study investigators and supported by the NIDDK. This paper was not prepared in collaboration with the investigators of the HEMO study and does not necessarily reflect the opinions or views of the HEMO study or the NIDDK.

Portions of this work were presented in abstract form at the American Society of Nephrology annual meeting in San Diego, 2012. 


\section{Disclosure Statement}

Dr. Mc Causland was supported by a Clinical Fellowship Grant from the National Kidney Foundation (2011-2013).

Dr. Brunelli has served as an advisor to Amgen, C.B. Fleet Company and Proctor \& Gamble. Since completing work on this study, Dr. Brunelli has become a full-time employee of DaVita Clinical Research. He has received speaking honoria from Fresenius Medical Care North America. His spouse is employed by Astra Zeneca. He was supported by DK079056 and Dr. Waikar was supported by DK093574, DK075941 and U01DK085660.

\section{References}

1 Bos WJ, Bruin S, van Olden RW, Keur I, Wesseling KH, Westerhof N, Krediet RT, Arisz LA: Cardiac and hemodynamic effects of hemodialysis and ultrafiltration. Am J Kidney Dis 2000;35:819-826.

-2 Boon D, van Montfrans GA, Koopman MG, Krediet RT, Bos WJ: Blood pressure response to uncomplicated hemodialysis: the importance of changes in stroke volume. Nephron Clin Pract 2004;96:c82-c87.

-3 Palmer BF, Henrich WL: Recent advances in the prevention and management of intradialytic hypotension. J Am Soc Nephrol 2008; 19 : 8-11.

4 Dheenan S, Henrich WL: Preventing dialysis hypotension: a comparison of usual protective maneuvers. Kidney Int 2001;59:1175-1181.

5 KDOQI: K/DOQI clinical practice guidelines for cardiovascular disease in dialysis patients. Am J Kidney Dis 2005;45:S1-S153.

6 Owen PJ, Priestman WS, Sigrist MK, Lambie SH, John SG, Chesterton LJ, McIntyre CW: Myocardial contractile function and intradialytic hypotension. Hemodial Int 2009; 13 : 293-300.

-7 Mizumasa T, Hirakata H, Yoshimitsu T, Hirakata E, Kubo M, Kashiwagi M, Tanaka $\mathrm{H}$, Kanai H, Fujimi S, Iida M: Dialysis-related hypotension as a cause of progressive frontal lobe atrophy in chronic hemodialysis patients: a 3-year prospective study. Nephron Clin Pract 2004;97:c23-c30.

8 Shoji T, Tsubakihara Y, Fujii M, Imai E: Hemodialysis-associated hypotension as an independent risk factor for two-year mortality in hemodialysis patients. Kidney Int 2004;66 1212-1220.

9 Kersh ES, Kronfield SJ, Unger A, Popper RW, Cantor S, Cohn K: Autonomic insufficiency in uremia as a cause of hemodialysis-induced hypotension. N Engl J Med 1974;290:650653.

10 Poldermans D, Man in 't Veld AJ, Rambaldi $R$, Van Den Meiracker AH, Van Den Dorpel MA, Rocchi G, Boersma E, Bax JJ, Weimar W, Roelandt JR, Zietse R: Cardiac evaluation in hypotension-prone and hypotension-resistant hemodialysis patients. Kidney Int 1999; 56:1905-1911.

-11 Kooman JP, Gladziwa U, Bocker G, van Bortel LM, van Hooff JP, Leunissen KM: Role of the venous system in hemodynamics during ultrafiltration and bicarbonate dialysis. Kidney Int 1992;42:718-726.
12 Song JH, Park GH, Lee SY, Lee SW, Kim MJ Effect of sodium balance and the combination of ultrafiltration profile during sodium profiling hemodialysis on the maintenance of the quality of dialysis and sodium and fluid balances. J Am Soc Nephrol 2005;16:237246.

13 Maynard JC, Cruz C, Kleerekoper M, Levin NW: Blood pressure response to changes in serum ionized calcium during hemodialysis. Ann Intern Med 1986;104:358-361.

14 Depner TA: Assessing adequacy of hemodialysis: urea modeling. Kidney Int 1994;45: 1522-1535.

15 Canzanello VJ, Hylander-Rossner B, Sands RE, Morgan TM, Jordan J, Burkart JM: Comparison of $50 \%$ dextrose water, $25 \%$ mannitol, and $23.5 \%$ saline for the treatment of hemodialysis-associated muscle cramps. ASAIO Trans 1991;37:649-652.

16 Eknoyan G, Beck GJ, Cheung AK, Daugirdas JT, Greene T, Kusek JW, Allon M, Bailey J, Delmez JA, Depner TA, Dwyer JT, Levey AS, Levin NW, Milford E, Ornt DB, Rocco MV, Schulman G, Schwab SJ, Teehan BP, Toto R: Effect of dialysis dose and membrane flux in maintenance hemodialysis. N Engl J Med 2002;347:2010-2019.

17 Greene T, Beck GJ, Gassman JJ, Gotch FA, Kusek JW, Levey AS, Levin NW, Schulman G, Eknoyan G: Design and statistical issues of the hemodialysis (HEMO) study. Control Clin Trials 2000;21:502-525.

18 Leypoldt JK, Cheung AK, Agodoa LY, Daugirdas JT, Greene T, Keshaviah PR: Hemodialyzer mass transfer-area coefficients for urea increase at high dialysate flow rates. The hemodialysis (HEMO) study. Kidney Int 1997; 51:2013-2017.

19 Miskulin DC, Athienites NV, Yan G, Martin AA, Ornt DB, Kusek JW, Meyer KB, Levey AS: Comorbidity assessment using the index of coexistent diseases in a multicenter clinical trial. Kidney Int 2001;60:1498-1510.

20 Zeger SL, Liang KY: Longitudinal data analysis for discrete and continuous outcomes. Biometrics 1986;42:121-130.

21 Liu T, Liang KV, Rosenbaum A, Stephenson R, Pike F, Weissfeld L, Unruh ML: Peripheral vascular disease severity impacts health outcomes and health-related quality of life in maintenance hemodialysis patients in the HEMO study. Nephrol Dial Transplant 2012; 27:2929-2936.
22 Lilley JJ, Golden J, Stone RA: Adrenergic regulation of blood pressure in chronic renal failure. J Clin Invest 1976;57:1190-1200.

23 Schroeder KL, Sallustio JE, Ross EA: Continuous haematocrit monitoring during intradialytic hypotension: precipitous decline in plasma refill rates. Nephrol Dial Transplant 2004; 19:652-656.

24 Tomita M, Malhotra D, Dheenan S, Shapiro JI, Henrich WL, Santoro TJ: A potential role for immune activation in hemodialysis hypotension. Ren Fail 2001;23:637-649.

-25 Arieff AI, Massry SG, Barrientos A, Kleeman CR: Brain water and electrolyte metabolism in uremia: effects of slow and rapid hemodialysis. Kidney Int 1973;4:177-187.

26 Pappius HM, Oh JH, Dossetor JB: The effects of rapid hemodialysis on brain tissues and cerebrospinal fluid of dogs. Can J Physiol Pharmacol 1967;45:129-147.

27 Kennedy AC, Linton AL, Eaton JC: Urea levels in cerebrospinal fluid after haemodialysis. Lancet 1962;1:410-411.

28 Mann H, Stiller S: Urea, sodium, and water changes in profiling dialysis. Nephrol Dial Transplant 1996;11(suppl 8):10-15.

29 Patel N, Dalal P, Panesar M: Dialysis disequilibrium syndrome: a narrative review. Semin Dial 2008;21:493-498.

30 Henrich WL, Woodard TD, Blachley JD, Gomez-Sanchez C, Pettinger W, Cronin RE: Role of osmolality in blood pressure stability after dialysis and ultrafiltration. Kidney Int 1980;18:480-488.

-31 Rodrigo F, Shideman J, McHugh R, Buselmeier T, Kjellstrand C: Osmolality changes during hemodialysis. Natural history, clinical correlations, and influence of dialysate glucose and intravenous mannitol. Ann Intern Med 1977;86:554-561.

32 Port FK, Johnson WJ, Klass DW: Prevention of dialysis disequilibrium syndrome by use of high sodium concentration in the dialysate. Kidney Int 1973;3:327-333.

33 Locatelli F, Covic A, Chazot C, Leunissen K, Luno J, Yaqoob M: Optimal composition of the dialysate, with emphasis on its influence on blood pressure. Nephrol Dial Transplant 2004;19:785-796.

-34 Sang GL, Kovithavongs C, Ulan R, Kjellstrand $\mathrm{CM}$ : Sodium ramping in hemodialysis: a study of beneficial and adverse effects. Am J Kidney Dis 1997;29:669-677. 
35 Locatelli F, Stefoni S, Petitclerc T, Coli L, Di Filippo S, Andrulli S, Fumeron C, Frasca GM, Sagripanti S, Savoldi S, Serra A, Stallone C, Aucella F, Gesuete A, Scarlatella A, Quarello F, Mesiano P, Ahrenholz P, Winkler R, Mandart L, Fort J, Tielemans C, Navino C: Effect of a plasma sodium biofeedback system applied to HFR on the intradialytic cardiovascular stability. Results from a randomized controlled study. Nephrol Dial Transplant 2012; 2012:4.

36 Shimizu K, Kurosawa T, Sanjo T: Effect of hyperosmolality on vasopressin secretion in intradialytic hypotension: a mechanistic study. Am J Kidney Dis 2008;52:294-304.
37 Mc Causland FR, Prior LM, Heher E, Waikar SS: Preservation of blood pressure stability with hypertonic mannitol during hemodialysis initiation. Am J Nephrol 2012;36:168-174.

38 van der Zee S, Thompson A, Zimmerman R, Lin J, Huan Y, Braskett M, Sciacca RR, Landry DW, Oliver JA: Vasopressin administration facilitates fluid removal during hemodialysis. Kidney Int 2007;71:318-324.

39 Chang TI, Paik J, Greene T, Desai M, Bech F, Cheung AK, Chertow GM: Intradialytic hypotension and vascular access thrombosis. J Am Soc Nephrol 2011;22:1526-1533.
0 McIntyre CW: Haemodialysis-induced myocardial stunning in chronic kidney disease - a new aspect of cardiovascular disease. Blood Purif 2010;29:105-110.

41 Flanigan M: Dialysate composition and hemodialysis hypertension. Semin Dial 2004;17: 279-283.

42 Greene T, Daugirdas J, Depner T, Allon M, Beck G, Chumlea C, Delmez J, Gotch F, Kusek JW, Levin N, Owen W, Schulman G, Star R, Toto R, Eknoyan G: Association of achieved dialysis dose with mortality in the hemodialysis study: an example of 'dose-targeting bias'. J Am Soc Nephrol 2005;16:3371-3380. 